\title{
Comunicación Política en tiempos de Nueva Cultura Política
}

\section{Political Communication in times of New Political Culture}

\section{Comunicação política em tempos de nova cultura política}

\author{
Xabier Barandiarán \\ Profesor (Universidad de Deusto) \\ https://orcid.org/0000-0002-7557-9331 \\ España \\ Alfonso Unceta \\ Catedrático (Universidad del País Vasco) \\ Director de Sinnergiak Social Innovation \\ https://orcid.org/0000-0003-2886-6204 \\ España \\ Simón Peña \\ Profesor (Universidad del País Vasco) \\ https://orcid.org/0000-0003-2080-3241 \\ España
}

Fecha de recepción: 8 de marzo de 2019

Fecha de revisión: 22 de abril de 2019

Fecha de aceptación: 13 de julio de 2019

Fecha de publicación: 1 de enero de 2020

Para citar este artículo: Barandiarán, X., Unceta, A. y Peña, S. (2020). Comunicación Política en tiempos de Nueva Cultura Política, Icono 14, 18 (1), 256-282. doi: 10.7195/ri14. v18i1.1382 


\section{INNOVACIÓN TEÓRICA}

\section{Resumen}

El proceso de globalización y sus manifiestos efectos comunicativos, culturales y políticos están afectando crucialmente a dos conceptos relativamente jóvenes en la investigación académica, en concreto, la Comunicación Política y la Cultura Política. La relación entre estos conceptos es cada vez más interdependiente pues las herramientas y los lenguajes de la Comunicación Politica influyen decisivamente en la conformación de la Nueva Cultura Política y, a su vez, la actividad de la ciudadanía a través de las herramientas comunicativas digitales está condicionando la generación de contenidos y la creación de los discursos que toman cuerpo en forma de Comunicación Política.

Creemos que para entender las razones de esta relación es necesario establecer, por un lado, las características de la Nueva Cultura Política y, por la otra, la manera en que las posibilidades que ofrece la tecnología transforman la generación y la transmisión de la Comunicación Política. Posiblemente lo más novedoso de esta relación es que tiene carácter bidireccional, alterando los roles clásicos del proceso comunicativo que distinguía nítidamente entre emisores y receptores. Novedad que constituye una evidencia global y se deja sentir de manera muy similar en el conjunto de las democracias representativas occidentales.

Este trabajo se esfuerza en describir y analizar este escenario emergente que ha tomado cuerpo como otra de las transformaciones que están aconteciendo en las sociedades avanzadas, y en identificar algunas tendencias que, en todo caso, están sometidas al cambio vertiginoso propio de nuestro tiempo.

\section{Palabras clave}

Cultura Política; Desafección; Participación; Comunicación Política; Tecnologías Digitales; Lenguajes Comunicativos 


\section{Abstract}

The process of globalisation and its clear communicative, cultural and political effects are greatly affecting two relatively new concepts in academic research, specifically Political Communication and Political Culture. The relationship between these concepts is increasingly interdependent as the tools and languages of Political Communication strongly influence the formation of the New Political Culture, and in turn, citizen activity through digital communication tools is conditioning the generation of content and the creation of discourse which take shape in the form of Political Communication.

We believe that to understand the reasons for this relationship it is necessary to establish, firstly, the characteristics of the New Political Culture, and secondly, the way in which the possibilities offered by technology transform the generation and transmission of Political Communication. Possibly the newest aspect of this relationship is that it has a two-way nature, altering the classic roles of the communication process that clearly distinguishes transmitters and receivers. This new aspect constitutes global evidence and is felt in a very similar way in all western representative democracies.

This work endeavours to describe and analyse this emerging scenario which has taken shape as another of the transformations which are occurring in advanced societies, and to identify some trends which, in all cases, are subject to the accelerated change of our time.

\section{Key Words}

Political Culture; Disaffection; Participation; Political Communication; Digital Technologies; Communicative Languages 


\section{INNOVACIÓN TEÓRICA}

\section{Resumo}

0 processo de globalização e os seus manifestos efeitos comunicativos, culturais e políticos estão a afetar crucialmente dois conceitos relativamente jovens na investigação académica, em concreto, a Comunicação Politica e a Cultura Política. A relação entre estes conceitos é cada vez mais interdependente, pois as ferramentas $e$ as linguagens da Comunicação Política influenciam decisivamente na formação da Nova Cultura Política e, por sua vez, a atividade da cidadania através das ferramentas comunicativas digitais está a condicionar a geração de conteúdos e a criação dos discursos que ganham forma como Comunicação Política.

Acreditamos que, para entender as razões desta relação, é necessário estabelecer, por um lado, as características da Nova Cultura Política e, por outro, a maneira como as possibilidades que oferece a tecnologia transformam a geração e a transmissão da Comunicação Política. Possivelmente a novidade desta relação é ter caráter bidirecional, alterando os papéis clássicos do processo comunicativo que distinguia nitidamente entre emissores e recetores. Novidade que consiste numa evidência global e que se deixa sentir de maneira muito semelhante no conjunto das democracias representativas ocidentais.

Este trabalho esforça-se por descrever e analisar este cenário emergente que ganhou corpo como outra das transformações que estão a acontecer nas sociedades avançadas, e em identificar algumas tendências que, em todo o caso, estão sujeitas à mudança vertiginosa própria do nosso tempo.

\section{Palavras chave}

Cultura Politica; Desafeição; Participação; Comunicação Política; Tecnologias Digitais; Linguagens Comunicativas 


\section{Introducción}

La noción de cultura política ha cumplido ya casi cuarenta años desde que comenzó a emplearse con cierta asiduidad en los estudios orientados a comprender la manera en que la ciudadanía establece su relación con la política.

Uno de los precursores del término, Lechner (1990; 1996), ha analizado en varios trabajos la trayectoria que los estudios sobre cultura política han dibujado durante estos años y los diferentes focos de interés que han ido paulatinamente abordando.

De acuerdo con Lechner (1990), en una primera fase, los estudios sobre cultura política pusieron el foco en las actitudes, valores y creencias de la ciudadanía (Almond y Verba, 1989), para orientarse, en una segunda fase, a analizar el impacto atribuido a la globalización de la cultura y la comunicación en la conformación de la cultura política (Castells, 2009).

La fase actual, la tercera fase, es especialmente compleja pues se advierte la confluencia de dos fenómenos. El primero de ellos, la profunda crisis de los sistemas democráticos representativos (Castells, 2017) algunas de cuyas causas analizaremos más adelante. El segundo, la penetración generalizada de mensajes comunicativos a través de los instrumentos tecnológicos digitales disponibles, y muy especialmente a través de internet (Chaves-Montero, 2017). Por descontado que nos estamos refiriendo a mensajes comunicativos relacionados con la política de una manera más o menos explícita.

De tal forma que, hoy como nunca, la comunicación política ha pasado a jugar un papel estratégico en la relación de la ciudadanía con la política, es decir, en la formación de la cultura política, en la manera en que la ciudadanía percibe, valora y juzga la política. Se trata de un fenómeno que afecta al conjunto de las democracias representativas de los sociedades avanzadas y que contribuye decisivamente a la crisis de la cultura política tradicional o vieja cultura política (Jurado, 2015). 


\section{INNOVACIÓN TEÓRICA}

Como bien señala Gil Calvo (2018),

la crisis de la democracia actual es un problema político para el que debemos investigar cuáles son sus responsables políticos, y solo contamos con dos tipos de agentes de quien sospechar: la clase política y la clase mediática. (...) De un lado la hipótesis A, planteada por los autores que interpretan el problema como "mediatización" de la política, en la que son los "medios" los que ocupan la variable independiente como "culpables" de causar la crisis de la democracia. Y, del otro, la hipótesis B, de aquellos otros que atribuyen su causa a la "politización" de los medios, en cuyo caso la variable independiente es la "clase política", como responsable última de la degradación electoralista de la democracia (p. 218-219).

En las líneas que siguen no pretendemos posicionarnos del lado de una u otra de las hipótesis planteadas por Gil Calvo (2018). Pero sí contextualizar y explicar la manera en que "nueva cultura política" y "comunicación política" se relacionan, para tratar de entender el alcance y las consecuencias de esta relación.

Para ello, en la Primera Parte de este artículo nos ocuparemos del significado de la "nueva cultura política", sus causas, algunas de sus expresiones y sus consecuencias, mientras en la Segunda Parte analizaremos de qué manera los nuevos instrumentos y contenidos de la "comunicación política" afectan a la puesta en cuestión del escenario clásico en el que se ha venido desenvolviendo la vieja cultura política.

\section{Primera Parte: La nueva cultura política}

\section{El cuestionamiento de la política tradicional}

La nueva cultura política es la expresión de la ausencia de categorías sociales, culturales e ideológicas de los problemas tradicionales de la política formulados en clave de izquierda y derecha. Ello confirma las sospechas de distintos autores sobre la realidad institucional de las sociedades post-industriales (Touraine, 1971; Bell, 1994). 
La nueva cultura política presenta también elementos de diferenciación con respecto a las viejas estructuras de acción y representación políticas propias de los sistemas democráticos clásicos. Siguiendo a Clark e Inglehart (1998) entre tales elementos de diferenciación cabe destacar los siguientes:

- El alto porcentaje de votantes que en la actualidad no se identifican con ningún partido político.

- El surgimiento de líderes políticos que rompen con los programas clásicos establecidos por sus propios partidos.

- El distanciamiento de la ciudadanía respecto de los asuntos oficiales de la vida pública.

- El sentimiento negativo de una parte de la ciudadanía hacia la política, traducido en muestras de descontento más o menos exacerbadas (Ganuza y Font, 2018).

Pero, ¿Cuáles son las razones de esta evolución?

Una primera razón relevante es la que tiene que ver con la forma en que los ciudadanos consumen (Bauman, 2007) el hecho político (escenarios de producción y consumo audiovisuales y digitales), que la mayoría de las veces no hace sino reproducir el debate entre los actores políticos, un debate centrado en la disputa de los titulares.

Una segunda razón igualmente relevante es la relacionada con el desencantamiento ciudadano con la política, debido en gran medida al incumplimiento de los axiomas en los que se fundamentaba la confianza en los sistemas democráticos representativos.

De acuerdo con Simone (2016), los "axiomas de la democracia" son aquellos en los que se han basado los derechos y las libertades de la ciudadanía en las democracias representativas (trabajo, salud, educación, igualdad, libertad de expresión), y cuya protección quedaba a cargo de la clase política. 


\section{INNOVACIÓN TEÓRICA}

En torno a tales axiomas se estabilizaron los discursos y las prácticas políticas que dieron lugar a que lo que se conoce como "vieja cultura política" haya tenido un grado de estabilidad y aceptación suficiente durante varias décadas. De hecho, el grado de legitimidad de esta vieja cultura política había conocido escasas oscilaciones a lo largo de las últimas décadas.

Sin embargo, en un periodo de tiempo relativamente breve, distintas causas han acelerado el cuestionamiento de esa forma tradicional de entender y practicar la política, por una parte, y de legitimarla, por la otra. Para comprender este giro vamos a mencionar dos grandes cuestiones que, a nuestro entender, explican buena parte de la crisis de las democracias representativas provocada por el cuestionamiento de sectores cada vez más amplios y diversos de la población:

- El impacto económico y consecuentemente social de la globalización y sus consecuencias.

- El sentimiento de amenaza a estructuras identitarias bastante consolidadas en nuestras sociedades.

\section{El impacto económico y social de la globalización}

La globalización inaugura una etapa que sucede a la hegemonía del sistema productivo industrial y que se caracteriza por una economía basada en los servicios, la innovación y el conocimiento. Una economía dirigida por el capitalismo financiero y marcada por la "posorganización" (Bell, 1994; Lash y Urry, 1998).

Nos referimos a una economía constantemente condicionada y afectada por los avances y la supremacía tecnológica que, entre otros efectos, ha provocado la "pérdida de centralidad y estabilidad del trabajo como elemento central de estructuración social" (Subirats, 2010).

Una economía que ha generado cada vez más desigualdad y que ha ampliado muy significativamente la nómina de desfavorecidos, marginados y excluidos. Una economía que ha generado mucho rechazo entre sectores muy diversos de la población. 
¿Cómo se puede admirar o respetar un modo de hacer que ha inventado la economía-ficción basada en la ingeniería financiera, que ha creado sofisticadas burbujas de <éter económico> que han arruinado a tantos millones de personas mientras unos pocos conseguían millones de dólares de beneficio personal? ( $\mathrm{Gu}$ tiérrez Conde, 2018, p. 341-342).

La globalización genera problemas que los estados se muestran incapaces de resolver, entre otras razones, porque la naturaleza de tales problemas desborda las fronteras territoriales. De ahí que buena parte de la ciudadanía vaya interiorizando una sensación creciente de incertidumbre e inseguridad.

A su vez, los políticos de las democracias representativas se muestran incapaces de apaciguar tales incertidumbres. Es más, en muchos casos, las acrecientan con lenguajes apocalípticos. En uno u otro supuesto, desde la perspectiva de la ciudadanía, la sensación de desprotección es bastante generalizada.

\section{Identidades amenazadas}

Las identidades producen, por una parte, sentido de vida y, por otra, operan como refugios frente a potenciales amenazas, supuestas o reales (Tajel, 1982). Sin embargo, es una evidencia que en sociedades complejas el debilitamiento de las identidades, o bien su fragmentación, es un fenómeno habitual.

Hoy día es un hecho que, en nuestros entornos existe un número muy importante de personas y grupos que sienten amenazada su identidad. Ello es debido en gran medida a distintos factores que pueden potencialmente erosionar las bases identitarias sean estas de carácter político, étnico, lingüístico, profesional, o de otro tipo.

Relacionado con lo anterior se están incrementando las actitudes y los comportamientos de repliegue y rechazo ante amenazas supuestas o reales. Buenos exponentes de ello son la radicalización de los sentimientos nacionalistas, del fundamentalismo de base religiosa o el incremento de la xenofobia. 


\section{INNOVACIÓN TEÓRICA}

Sin duda, el fenómeno de la emigración está teniendo gran influencia en la extensión de este tipo de actitudes y comportamientos. Es evidente que la emigración, amenaza para algunos, oportunidad para otros, produce sentimientos de hostilidad y rechazo en colectivos social y numéricamente significativos.

Al respecto, Mounk (2018) afirma que,

desde el momento en que comenzó esa llegada masiva de inmigrantes a sociedades que se definían a sí mismas por una cultura y un origen étnico compartidos, la tensión entre teoría y práctica se fue volviendo cada vez más explosiva. De ahí que seguramente no deba sorprendernos que, en las últimas décadas, haya aumentado tan rápido el apoyo a una serie de fuerzas políticas radicalmente opuestas a la inmigración (p. 171).

Evidentemente estos sentimientos y comportamientos son más visibles entre la población que convive socio-espacialmente con la población inmigrante, pero tienden a extenderse a sectores más amplios de la ciudadanía por efecto de discursos y relatos que han encontrado en la gran variedad de estructuras comunicativas disponibles en un terreno abonado para su difusión a gran escala.

\section{3. ¿Cómo identificar la nueva cultura política?}

La nueva cultura política presenta elementos de diferenciación con respecto a las viejas estructuras de acción y representación de los procesos de modernización democrática clásicos (Clark e Inglehart, 1998).

Estamos asistiendo a un proceso generalizado de pluralización y desdramatización (en el sentido fuerte del término) de las formas de vivir la relación con la política y los acontecimientos políticos. La pluralización a que nos referimos está basada, en muchos casos, en intereses de carácter más cultural que político que genera comunidades articuladas a través de redes y que, en bastantes ocasiones, son poco visibles socialmente. 
Puede decirse que este abandono de la vivencia intensa del hecho político se ha ido generalizando y tiene también un componente sentimental. Como señala Innerarity (2018), "hay decepcionados por todas partes y por muy diversos motivos, frecuentemente contradictorios, en la derecha y en la izquierda, a los que ha decepcionado el pueblo o se sienten traicionados por las élites" (p. 53).

Vamos a presentar a continuación algunos indicadores de esta realidad.

\section{La potencialidad de los movimientos sociales}

Están cobrando cada vez más protagonismo movimientos sociales (ecologistas, pacifistas, feministas etc.) que, a través de mecanismos de acción colectiva buscan fortalecer la democracia, la descentralización, el respeto a la diversidad y a la libertad individual.

El caso del feminismo está siendo especialmente llamativo en los últimos años. El feminismo es la voz de una de las nuevas dimensiones de la vida social que quiere ser escuchada para poder tener un espacio reconocido en la sociedad. Se instala en la oposición al orden establecido para las mujeres por el sistema cultural hegemónico, reivindicando el derecho a tener un espacio de igualdad respecto a los hombres, en el cual las mujeres tengan mayor autonomía, mayor poder social y prestigio (Bullén, 2017, p. 58).

Tampoco debemos perder de vista las movilizaciones protagonizadas por muchas personas jóvenes que han traspasado fronteras nacionales y han tenido gran impacto en países como España. Son indicativas de un movimiento que puede que no sea coyuntural y que cristalice en un sentimiento/identidad de grupo social cuyos intereses (acceso a recursos públicos por ejemplo) colisionan con los de otros grupos sociales (el caso de las reivindicaciones de las personas jubiladas).

En esta línea debemos destacar que una idea bastante extendida entre muchos jóvenes es que tienen que sortear una y otra vez barreras que condicionan severamente sus expectativas y su trayectoria vital. 


\section{INNOVACIÓN TEÓRICA}

Los jóvenes se enfrentan a un muro que les impide construir su futuro. Los ladrillos que forman este muro son la precariedad, la falta de oportunidades, 0 la ausencia de posibilidades para emanciparse y construir un hogar. También la ignorancia, la falta de interés (en el mejor de los casos) o la corrupción (en el peor) de nuestros servidores públicos a la hora de buscar soluciones (Politikon, 2017, p.12).

\section{Desafección ciudadana con respecto a la política}

De todos es conocido el alto porcentaje de votantes que en la actualidad no se identifica con ningún partido político y el cada vez más evidente comportamiento de candidatos y líderes políticos que rompen con los programas clásicos establecidos por sus propios partidos en un intento por interesar a colectivos alejados de la política.

En opinión de Simón (2018), “los ciudadanos dan más la espalda a los partidos porque el mundo ha cambiado y no son tan relevantes, o porque los partidos mismos, al vaciarse de ideología, les interesan menos" (p.71). Una tendencia que el propio Simón (2018) ha denominado "la bancarrota del sistema de partidos" ( $\mathrm{p}$. 57).

Lo cierto es que la política es percibida por buena parte de la ciudadanía como una actividad ajena lo que produce un distanciamiento ciudadano cada vez mayor con respecto a la actividad política convencional (Mair, 2013). Como señala Innerarity (2018), "el actual paisaje político se ha llenado de una decepción generalizada que ya no se refiere a algo concreto sino a una situación en general" (p. 9).

Una de las razones de este distanciamiento es que los actores políticos tienen vida propia por lo general ajena a una gran mayoría de los ciudadanos con la que se relacionan esporádicamente. Se trata de un proceso fuerte de privatización de la política. 
Los que creen que viven en el escaparate de la política, que suelen ser los que se creen que están escribiendo la historia, casi nunca son generosos y no suelen hacer el esfuerzo de explicar el motor escondido que mueve las cosas" (Del Olmo, 2018, p. 9).

De tal manera que los políticos profesionales se han socializado en un ecosistema cerrado, entienden su función de una manera excesivamente vertical e interiorizan que la proyección mediática es una de las claves del éxito. Así, están más preocupados por su impacto mediático (con o sin contenido), algo que tiene que ver con la simple propaganda y no con la resolución de los problemas de la ciudadanía. La agenda política es la mejor expresión de la brecha entre la política (los políticos) y la sociedad (la ciudadanía).

\section{La política de las emociones: el populismo}

La relación entre políticos y ciudadanía que venimos de describir ha creado un notable vacío que el populismo está aprovechando para ofrecer un conjunto de recetas que contienen soluciones simples y rápidas a los problemas de nuestro tiempo. Pero como bien señala Mounk (2018),

la disposición de los líderes populistas a ofrecer soluciones tan simples que no pueden funcionar de ningún modo es muy peligrosa. Cuando llegan al poder, sus políticas tienden más bien a exacerbar los problemas mismos que suscitaron las iras populares que los llevaron al gobierno (p.44).

Es la política de las emociones que está siendo practicada con notable éxito por personas y partidos políticos.

Innerarity (2017) se refiere acertadamente a este componente emocional bajo el término de "sociedades exasperadas" (p.53). Ciudadanos que se sienten vulnerables, atenazados por el miedo convenientemente generado a distintos tipos de amenazas, impacientes por la falta de soluciones a los problemas que perciben. 


\section{INNOVACIÓN TEÓRICA}

Las emociones y los sentimientos se imponen al pensamiento racional.

Tenemos un paisaje colectivo en el que se contagian y realimentan los afectos caóticos de un precariado ansioso, consumidores compulsivos, sociedades en alerta máxima, mercados histéricos, amenazas ubicuas y ciudadanos desconfiados" (Innerarity, 2017, p. 60).

El pensamiento lógico queda enfrentado a las situaciones emocionales. Como bien señala Del Olmo (2018), "los límites de la razón están lejos de ser un descubrimiento nuevo, y la importancia de las emociones ha expandido la política postmoderna desde las fronteras de los gobiernos hasta las de la oposición" (p.108).

Pero, de hecho, la racionalidad y la burocratización alejan a la vieja política de las soluciones que se le reclaman. La política convencional no es capaz de moverse adecuadamente por esa geografía de la emoción (Del 0lmo, 2018) todo lo contrario que la propuesta populista.

El componente emocional está en la base del ascenso del populismo. Como indica Lassalle (2017),

para entender el proceso populista hay que hurgar en la estrcutura emocional de las sociedades occidentales y en el colapso de la Ilustración. (...) Se trata de reventar -mediante el empleo de sucesivas minas emocionales- la arquitectura y la democracia liberal surgida del siglo XVIII" (p.17-18).

El crecimiento y la extensión del fenómeno populista se comprenden mejor si consideramos el factor tecnológico, porque las redes sociales se han convertido en un terreno de juego habitual y, en ocasiones crucial para la acción política. Así, como vamos a fundamentar en la Segunda Parte de este artículo, en la extensión de esta geografía de la emoción, las tecnologías al servicio de la comunicación política juegan un papel determinante, transformando incluso el propio concepto de Comunicación Política. 


\section{Segunda Parte: La Comunicación Política}

\section{La sofisticación de la Comunicación Política}

La comunicación política es un quehacer orientado a la generación de creencias y opiniones sobre los asuntos políticos. En tal sentido, el destinatario principal de la comunicación política es la ciudadanía y los emisores principales de información son los propios políticos.

Sin embargo, lo que caracteriza a la etapa actual de la comunicación política es que la ciudadanía se transforma en un agente activo y crítico que usa la tecnología como elemento para la toma de decisiones políticas, frente a modelos en los que la participación directa lo era solo en apariencia (Blumler y Kavanagh, 2000).

Siguiendo a Blumler y Kavanagh (2000), la primera etapa de la comunicación política -iniciada tras el final de la Segunda Guerra Mundial- se había caracterizado por la subordinación de buena parte de la comunicación a valores e instituciones políticas estables y fuertes mientras que, en la segunda etapa, una comunicación política crecientemente profesionalizada había adaptado los contenidos al formato televisivo para seducir a un electorado cuya opinión podía resultar variable.

Por el contrario, en la tercera etapa -caracterizada por la sobreabundancia de información- Blumler y Kavanagh (2000) identifican cinco tendencias que van progresivamente consolidándose:

- Mayor necesidad de profesionalización.

- Mayor competencia.

- Aparición de un populismo antielitista.

- Diversificación centrífuga.

- Cambios en la forma en la que las personas perciben la política. 


\section{INNOVACIÓN TEÓRICA}

Esta tercera etapa a la que nos estamos refiriendo, se ha visto esencialmente condicionada por la irrupción de la denominada web 2.0, que ha traído consigo la configuración de una nueva esfera pública de participación ciudadana. Ello ha dado lugar, por ejemplo, a movilizaciones instantáneas en torno a determinados acontecimientos de índole política con un componente de reivindicación o protesta que Rheingold (2004) define con el término "multitudes inteligentes".

Asimismo, en esta etapa, el uso de las nuevas tecnologías ha contribuido al desarrollo del ideal del modelo de Gobierno Abierto, entendido como un modelo de comunicación horizontal (Calderón, 2011) o, si se prefiere, una conversación de las instituciones con los ciudadanos sobre las cuestiones políticas, lo que constituye un síntoma de buena salud de la cultura democrática (Warner, Turner y Hawthorne, 2013).

Sin embargo, varias son las sombras que se expanden sobre el uso de las nuevas tecnologías en la comunicación política. Vamos a enumerar algunas de ellas:

- El miedo a la crítica y el deseo de control de la comunicación política por parte de los emisores (Macnamara, Safinofsky y Beattie, 2012).

- La baja participación en muchos de los nuevos canales habilitados, especialmente en las redes sociales, con lo que no parecen reconducirse los problemas de movilización del electorado desencantado (Warner, Turner y Hawthome, 2013).

- La sospecha de que una creciente exposición a este tipo de mecanismos comunicativos no garantiza una mayor participación de la ciudadanía en la política (Tuviera-Puigbó, 2009).

- La dificultad de buena parte de la clase política de hacerse con el lenguaje propio de los mecanismos comunicativos digitales, y la permanencia del lenguaje de uso en medios audiovisuales como la televisión (Del Rey, 2012).

- El rechazo de muchos políticos a los nuevos canales comunicativos, bien sea por una cuestión de brecha generacional o por escepticismo sobre los beneficios del uso de estos dispositivos tecnológicos (Ureña, 2011). 
En definitiva, a pesar de que las redes sociales ofrecen mayores facilidades para contactar directamente, otorgar visibilidad a los participantes, fomentar los debates o incluso alertar sobre posibles conflictos, la clase política hace un uso bastante testimonial de las mismas y solamente en algunos casos se diseñan estrategias activas y permanentes de comunicación política a través de estos mecanismos (Túnez y Sixto, 2011).

En el marco de esta contribución ambivalente de los nuevos sistemas digitales a la comunicación política, Ainsworth, Hardy y Harley (2005) subrayan los tres posicionamientos que se observan en relación a los beneficios y los límites del uso de estas tecnologías en la comunicación política:

- Quienes ponen en valor el potencial democrático del uso de la red, fundamentalmente en tanto facilitan la participación de sectores muy diversos, incluso de sectores tradicionalmente excluidos del diálogo político.

- Quienes enfatizan las discriminaciones que provoca su uso, derivadas de situaciones de género, clase o raza.

- Quienes hacen depender las potencialidades y los beneficios de la red del comportamiento de los usuarios, fundamentalmente en clave de comunidad y de responsabilidad social.

\section{Los riesgos de la Comunicación Política en el tiempo presente}

Ya hemos fundamentado que el uso de la tecnología digital está transformando el modo en que las informaciones se producen, distribuyen y reciben (Boczkowski y Anderson, 2017). Esta transformación está también influyendo en el comportamiento político de los ciudadanos, pero lo que no está claro es que contribuya a una mayor participación (Prior, 2007). Es decir, el incremento del número de noticias y comunicaciones en circulación y de las posibilidades de entretenimiento generalista, no necesariamente redunda en una mayor participación política. 


\section{INNOVACIÓN TEÓRICA}

Lo anterior mucho tiene que ver con que, paradójicamente, esta multiplicación de canales y contenidos informativos puede incrementar el riesgo de desinformación entre la ciudadanía. Ello se debe fundamentalmente a dos razones:

- Un mayor volumen de información no necesariamente genera una población mejor informada (Kampen y Snijkers, 2003).

- Al incremento de usuarios y actores presentes en los canales digitales ha acompañado un conjunto de prácticas interesadas que no se rigen por criterios informativos como la veracidad.

Muchos son los ejemplos de lo que se ha conocido como "noticias falsas", especialmente las evidencias de lo sucedido en la campaña en la que Donald Trump fue elegido presidente (Alcot y Gentzkow, 2017; Guess, Nihan y Reifler, 2018) o en el referéndum sobre el Brexit (Bastos y Mercea, 2017).

Según el Digital News Report (2018), la manipulación de las informaciones periodísticas para servir a unos determinados intereses políticos o económicos es la principal fuente de preocupación para los usuarios (77\%). Son los mismos usuarios los que opinan que son los medios informativos y los periodistas los que deben velar por la veracidad de las noticias (84\%), atribuyendo también a empresas tecnológicas (79\%) y gobiernos (72\%) una gran responsabilidad (Amoedo, Vara y Negredo, 2018).

Junto con el riesgo de desinformación o de una información poco veraz, la tercer etapa de la comunicación política debe enfrentar también el riesgo de la falta de pluralismo, bien por el "control" en redes sociales de determinados grupos de usuarios que comparten ideas y visiones sobre la realidad (Sunstein, 2017) o bien por la dificultad de algunos grupos de acceder en condiciones a estas plataformas digitales (Bakshy, Messing y Adamic, 2015).

Hermes (2006) concluye que el uso de las nuevas tecnologías orientado a la comunicación política no está logrando una comunicación a gran escala entre grandes grupos de personas sino que tiende a crear comunidades cerradas y de pequeño tamaño. 


\section{Tecnologías digitales y diálogo horizontal}

Una perspectiva diferente y complementaria con la presentada en el apartado anterior es la que pone el acento en la contribución de las tecnologías digitales al fomento del diálogo horizontal. Del Olmo (2018) señala acertadamente esta paradoja:

Por una parte, la red redobla las posibilidades de confundir y exige de la ciudadanía muchas más habilidades a la hora de distinguir los contenidos verdaderos y falsos. (...) Por la otra, el reordenamiento de la conversación pública, la alteración del formato comunicativo, destruye el orden el poder. El poder requiere que la comunicación transcurra en una sola dirección, de arriba abajo. Y hoy, con la aparición de la red social y la crisis de representación ya no somos meros receptores y consumidores pasivos de informaciones. (p.175-181).

Por eso, en una época en la que se constata el aumento de la desconfianza de la ciudadanía con respecto a la política, las posibilidades que ofrecen las tecnologías para expandir la comunicación política e incrementar la participación ciudadana, es uno de los aspectos que más interesan a analistas e investigadores (Kampen y Snijkers, 2003; Rheingold, 2004).

Lo relevante de esta capacidad potencial es que la cultura política que puede construirse a través de los medios digitales no puede entenderse exclusivamente en términos tecnológicos. Es decir, no se trata simplemente del acceso a la información a través de nuevos canales, se trata de algo más profundo.

Lo que estamos sugiriendo es que la capacidad potencial de los medios digitales aplicada a la comunicación política constituye todo un fenómeno cultural en la medida en que las audiencias y los usuarios vayan asumiendo un papel más dinámico. Es decir, que las audiencias y los usuarios se sientan capacitados y motivados para crear y difundir contenidos (Lewis, 2012).

Lo que queremos destacar es esta capacidad de los medios digitales para abrir diálogos de ida y vuelta, basados en el dinamismo de la ciudadanía, lo que puede incrementar la participación e incluso jugar un rol acelerador en la transformación 


\section{INNOVACIÓN TEÓRICA}

de las democracias representativas (Papacharissi, 2010). Bien es cierto que todo ello debe suceder en un escenario en el que la comunicación política es hoy más que nunca un claro exponente de la inmediatez teñida casi siempre de emoción. Inmediatez, y emoción son también palabras clave de la lógica comunicativa contemporánea.

Ahora bien, si bien los medios de comunicación han sido tradicionalmente los mediadores entre los líderes y el público, y han constituido el epicentro del debate público (Monzón, 1996), en la actualidad han perdido la exclusividad y la capacidad de monopolizar dicho debate.

Esto ha permitido que nuevos actores, como los propios partidos políticos y las instituciones, abran nuevos espacios en los que reclaman una presencia propia, pero el éxito de lo que Tuñez y Sixto (2011) denominan un "compromiso 2.0", no depende únicamente de sus actitudes, sino también de los usos que realice la ciudadanía de esas oportunidades.

Los estudios existentes demuestran una mayor correspondencia entre el uso de las tecnologías digitales y la participación en asuntos públicos, en especial entre los jóvenes (Arriagada y Schuster, 2011), lo que ofrece la posibilidad de promover nuevas prácticas cívicas que desafíen la concepción tradicional de la democracia participativa (Scherman, Arriagada y Valenzuela, 2012, p. 184).

En otras palabras, si bien parece que el uso de redes sociales tiene un impacto menor que el de los medios tradicionales en la predisposición a votar -sobre el que tiene más bien un carácter de refuerzo-, puede afirmarse que sus efectos más positivos provienen de las interacciones multidireccionales que promueven la participación política más allá del simple ejercicio de ir a votar (Navia y Ulriksen, 2017, p. 83).

\section{Conclusiones}

Este artículo no pretende ir más allá de los propios límites impuestos. Así, hemos aportado un conjunto de reflexiones, evidencias y tendencias que dan sobrada 
cuenta de que los actuales procesos de cambio y transformación social y tecnológica están transformando los mecanismos que conforman la cultura política y los formatos y contenidos de la comunicación política.

Cuestión distinta es que estemos en condiciones de añadir a lo anterior un apéndice empírico en el que poder contrastar algunas de las intuiciones que este trabajo sugiere. Si podemos avanzar que estamos inmersos en un trabajo sólido de contraste y verificación a través de una comunidad virtual que venimos monitorizando desde hace ya unos meses. Pero no pueden todavía avanzarse resultados fiables.

Lo que si podemos anticipar es que cualquier estudio empírico que trate de entender las consecuencias de las nuevas formas de comunicación política en el escenario abierto por la nueva cultura política, debe plantear una segmentación de la ciudadanía diferente y más compleja de las que tradicionalmente han venido utilizándose.

Entendemos que el enfoque apropiado para entender los efectos de las formas contemporáneas de comunicación política en el tipo de relación que la ciudadanía establece con la política, debe descansar en una aplicación específica de las teorías clásicas sobre actitudes, considerando muy especialmente la relación entre creencias, emociones y comportamientos.

Creemos que solamente a la luz de una segmentación que considere estos tres factores puede accederse a una comprensión más ajustada de la influencia que las nuevas formas de comunicación política están teniendo en los posicionamientos de la ciudadanía ante el hecho político.

Mientras tanto, lo que si estamos en condiciones de reafirmar es la intensa relación e interacción entre la comunicación política y la cultura política, dos conceptos cada vez más vinculados. Sobre la base de esta certeza queremos finalmente resaltar, a modo de sumario, los tres signos más visibles de este vínculo, y con ello las claves que contribuyen a ubicarlo y comprenderlo. 


\section{INNOVACIÓN TEÓRICA}

La compleja relación entre comunicación y política

La evolución y ampliación de las tecnologías digitales y por tanto de los medios al servicio de la comunicación política están creando un escenario complejo y condicionante para la conformación de la cultura política. Los medios convencionales anteriores a la expansión de las tecnologías digitales que difundían la información de manera unidireccional y vertical, cohabitan y se complementan con nuevas alternativas informativas y lenguajes comunicativos. Así, estos canales y lenguajes están condicionando muy significativamente la <dimensión cognitiva> de la cultura política, en un tiempo en el que, además, lo virtual puede llegar a tener más credibilidad que la propia realidad.

La comunicación y la política se necesitan

La relación de necesidad entre la comunicación y la política se comprende entendiendo los comportamientos y actitudes de tres agentes diferentes:

- Los actores políticos usan y abusan del universo mediático analógico y digital para difundir sus mensajes y canalizar las estrategias de marketing político y electoral, "en esta campaña continua que vivimos actualmente" (Gil Calvo, 2018, p. 199).

- Los medios de comunicación tienen la capacidad de dar visibilidad o no a los mensajes lanzados desde la política, apoyarlos o criticarlos. Por tanto, los medios tienen un rol activo en la formación de la opinión pública y en el desarrollo del proceso político (Eilders, 2000), es decir, en la configuración de la cultura política.

- El conjunto de ciudadanos bien en su condición más pasiva (espectadores) o más activa (actores), encuentran en ese universo mediático su principal alimento informativo sobre los asuntos políticos. Los ciudadanos precisan de los medios para seguir los asuntos públicos, recopilar información sobre temas y opiniones, informarse, formar opinión y participar del proceso político (Eilders, 2000). 
- La comunicación y la política: nuevos roles ciudadanos

Aprovechando las posibilidades que la innovación tecnológica brinda al diálogo horizontal, estamos asistiendo a un cierto grado de movilización social representada en el "activismo digital" (Morozov, 2017).

Este activismo digital ha abierto nuevos escenarios de participación en la esfera pública en los que la inmediatez de la información (veraz o ficticia), las facilidades para la interacción y la rapidez colectiva para emitir opiniones, han puesto de manifiesto la fragilidad del panorama político tradicional dando paso a una nueva cultura política que hoy se estructura sobre los cimentos de una baja confianza en las instituciones y la apatía política.

En este contexto, los nuevos canales y lenguajes de comunicación están siendo considerados como uno de los principales responsables de las actitudes negativas de los ciudadanos hacia el sistema político (Jorge y Miró, 2011 ).

Consecuentemente, al redefinirse y ampliarse los canales de comunicación política, se han reconfigurado los roles de emisor y receptor. Cada vez son más los receptores activos que se convierten en emisores de información y opinión política.

\section{Referencias bibliográficas}

Ainsworth, S., Hardy, C. y Harley, B. (2005). Online consultation: e-democracy and e-resistance in the case of the development gateway. Management Communication Quarterly, 19(1), 120-145. https://doi.org/10.1177/0893318905276562

Allcott, H. y Gentzkow, M. (2017). Social media and fake news in the 2016 election. Journal of economic perspectives, 31(2), 211-36. https://doi.org/10.1257/ jep.31.2.211

Almond, G. A. y Verba, S. (1989). The civic culture: Political attitudes and democracy in five nations. London: Sage Publications.

Amoedo, A., Vara, A. y Negredo, S. (2018). DigitalNewsReport.es 2018. Navarra: Universidad de Navarra. 


\section{INNOVACIÓN TEÓRICA}

Arriagada, A. y Schuster, M. (2011). Consumo de medios y participación ciudadana de los jóvenes chilenos. Cuadernos. Info, (22), 52-64. https://doi.org/10.7764/ cdi.22.87

Bakshy, E., Messing, S. y Adamic, L. A. (2015). Exposure to ideologically diverse news and opinion on Facebook. Science, 348(6239), 1130-1132. http://dx.doi. org/10.1126/science.aaa1160

Bastos, M. T. y Mercea, D. (2017). The Brexit botnet and user-generated hyperpartisan news. Social Science Computer Review, 37(1), 38-54. https:// doi.org/10.1177/0894439317734157

Bauman, Z. (2007). Vida de consumo. México, D.F.: Fondo de cultura económica.

Bell, D. (1994). El advenimiento de la Sociedad post-industrial. Madrid: Alianza Editorial.

Blumler, J. G. y Kavanagh, D. (2000). The third age of political communication: Influences and features. Political communication, 16(3), 209-230. https://doi. org/10.1080/105846099198596

Boczkowski, P. J. y Anderson, C. W. (2017). Remaking the news: Essays on the future of journalism scholarship in the digital age. Cambridge: MIT Press.

Bullen, Margaret. (2017). La antropología feminista: Aportaciones conceptuales para una epistemología participativa. En J. Martinez (Ed.) Participar desde los feminismos: Ausencias, expulsiones y resistencias (pp. 29-63). Barcelona: Icaria.

Calderón, C. (2011). ¿Qué es el gobierno abierto? Revista Evoca, (4), 5-9.

Casero-Ripollés, A. y Yeste, E. (2014). La comunicación política hoy: entre nuevos medios y viejas lógicas. Trípodos. Facultat de Comunicació $i$ Relacions Internacionals Blanquerna, (34).

Castells, M. (2009). Comunicación y poder. Madrid: Alianza Editorial.

Castells, M. (2017). Ruptura. La crisis de la democracia liberal. Madrid: Alianza Editorial.

Chaves-Montero, A. (Ed). (2017). Comunicación Política y Redes Sociales. Sevilla: Egregius Ediciones.

Clark, N. y Inglehart, R. (1998). The New Political Culture: Changing Dynamics of Support for the Welfare State and other Policies in Postindustrial Societies. En, T. Clark y V. Hoffman-Martinot, (eds), The new political culture (pp. 9-72). Boulder: Westview Press. 
Del Olmo, J. (2018). La política por otros medios. Madrid: La Catarata.

Del Rey Morató, J. (2008). Comunicación política, Internet y campañas electorales: de la teledemocracia a la ciberdemocracia. Madrid: Tecnos.

Eilders, C. (2000). Media as political actors? Issue focusing and selective emphasis in the German quality press. German Politics, 9(3), 181-206. https://doi. org/10.1080/09644000008404613

Ganuza, E. y Font, J. (2018). ¿Por qué la gente odia la política?: Cómo nos gustaría que se tomaran las decisiones políticas. Madrid: La Catarata.

Gil Calvo, E. (2018). Comunicación política. Madrid: La Catarata.

Guess, A., Nyhan, B. y Reifler, J. (2018). Selective exposure to misinformation: Evidence from the consumption of fake news during the 2016 US presidential campaign. European Research Council, 9. Recuperado de http://www.askforce.org/web/Fundamentalists/Guess-Selective-Exposure-to-MisinformationEvidence-Presidential-Campaign-2018.pdf

Gutiérrez Conde, J. (2018). Atrapados por el futuro. Madrid: Pirámide.

Hermes, J. (2006). Citizenship in the Age of the Internet. European Journal of Communication, 21(3), 295-309. https://doi.org/10.1177/0267323106066634 Innerarity, D. (2017). Política para perplejos. Barcelona: Galaxia Gutenberg.

Jorge, J. E. y Miró, E. M. (2011). Medios de comunicación, democracia y cultura política. Question, 1. Recuperado de http://sedici.unlp.edu.ar/ handle/10915/34423

Jurado, F. (2015). Nueva gramática Política: de la revolución en las comunicaciones al cambio de paradigma. Barcelona: Icaria.

Kampen, J. K. y Snijkers, K. (2003). E-democracy: A critical evaluation of the ultimate e-dream. Social Science Computer Review, 21(4), 491-496.

Lash, S. y Urry, J. (1998). Economía de signos y espacios: sobre el capitalismo de la posorganización. Buenos Aires: Amorrortu.

Lassalle, J. M. (2017). Contra el populismo. Cartografía de un populismo postmoderno. Barcelona: Editorial Debate.

Lechner, N. (1990). Los patios interiores de la democracia: subjetividad y política. México, D.F.: Fondo de Cultura Económica.

Lechner, N. (1996). ¿Por qué la política ya no es lo que fue? Leviatán: Revista de hechos e ideas, (63), 63-74. 


\section{INNOVACIÓN TEÓRICA}

Lewis, S. C. (2012). The tension between professional control and open participation: Journalism and its boundaries. Information, Communication \& Society, 15(6), 836-866. https://doi.org/10.1080/1369118X.2012.674150

Macnamara, J., Sakinofsky, P. y Beattie, J. (2012). E-electoral engagement: How governments use social media to engage voters. Australian Journal of Political Science, 47(4), 623-639. https://doi.org/10.1080/10361146.2012.731491

Mair, P. (2013). Gobernando el vacío. La banalización de la democracia occidental. Madrid.: Alianza Editorial.

Monzón, C. (1996). Opinión pública, comunicación y política. Madrid: Tecnos.

Morozov, E. (2017). El impacto del activismo digital en la política de la Post Guerra Fria en La Era de la Perplejidad. Repensar el Mundo que conocíamos. Madrid: BBVA. Recuperado de https://www.bbvaopenmind.com/articulos/el-impactodel-activismo-digital-en-la-politica-de-la-post-guerra-fria/

Mounk, Y. (2018). El pueblo contra la democracia. Barcelona: Paidós.

Navia, P. y Ulriksen, C. (2017). Tuiteo, luego voto. El efecto del consumo de medios de comunicación y uso de redes sociales en la participación electoral en Chile en 2009 y 2013. Cuadernos, Info, (40), 71-88. https://doi.org/10.7764/ cdi.40.1049

Papacharissi, Z. (2010). A Prívate Sphere: Democracy in a Digital Age. Cambridge: Polity.

Politikon. (2017). El muro Invisible. Barcelona: Editorial Debate.

Prior, M. (2007). Post-Broadcast Democracy: How Media Choice Increases Inequality in Political Involvement and Polarizes Election. New York: Cambridge University Press.

Rheingold, H. (2004). Multitudes inteligentes: La próxima revolución social. Barcelona: Gedisa.

Scherman, A., Arriagada, A. y Valenzuela, S. (2012) ¿Hacia una nueva ciudadanía multifuncional? Uso de medios digitales, redes sociales online y participación política. Revista Latinoamericana de Opinión Pública, 2, 159-185.

Simón, P. (2018). El príncipe moderno. Democracia, política y poder. Barcelona: Editorial Debate.

Simone, R. (2016). El Hada Democrática. Por qué la Democracia Fracasa. Madrid: Taurus. 
Subirats, J. (2010). Si la respuesta es gobernanza, ¿cuál es la pregunta? Factores de cambio en la política y en las políticas. EKONOMIAZ. Revista vasca de Economía, 74(02), 16-35. Recuperado de https://ddd.uab.cat/pub/artpub/2010/108181/ ekonomiaz_a2010n74p16.pdf

Sunstein, C. (2017). Republic. Divided Democracy in the Age of Social Media. Princeton: Princeton University Press.

Tajel, H. (1982). Social Identity and Intergroup Relations. Cambridge: Cambridge University Press.

Touraine, A. (1971). La Sociedad Post-Industrial. Barcelona: Ariel.

Turiera-Puigbò, T. (2009). Què està canviant l'ús d'internet en la manera de fer i comunicar l'acció política?. Quaderns del CAC, (33), 13-19.

Túñez, M. y Sixto, J. (2011). Redes sociales, política y Compromiso 2.0: La comunicación de los diputados españoles en Facebook. Revista Latina de comunicación social, (66), 210-234. Recuperado de http://www.revistalatinacs. org/11/art/930_Santiago/09_Tunez.html

Ureña, D. (2011). Decálogo para un candidato 2.0. Cuadernos de comunicación evoca, 4(29-33).

Warner, B. R., McGowen, S. T. y Hawthorne, J. (2012). Limbaugh's social media nightmare: Facebook and Twitter as spaces for political action. Journal of Radio \& Audio Media, 19(2), 257-275. https://doi.org/10.1080/19376529.20 12.722479

\section{(c) (1)}

Este obra está bajo una licencia de Creative Commons Reconocimiento 4.0 Internacional. 